\title{
Packaging Incomplete or Missing
}

National Cancer Institute

\section{Source}

National Cancer Institute. Packaging Incomplete or Missing. NCI Thesaurus. Code C62940.

Problem associated with the nonconformance to the device specifications due to incomplete or missing packaging that may compromise the device operation as intended. 